\title{
Effects of the new hadronic interaction models on the reconstruction of KASCADE-Grande observables
}

A. Gherghel-Lascu ${ }^{* 5}$, W.D. Apel ${ }^{1}$, J.C. Arteaga-Velázquez ${ }^{2}$, K. Bekk ${ }^{1}$, M. Bertaina ${ }^{3}$, J. Blümer ${ }^{1,4}$, H. Bozdog ${ }^{1}$, I.M. Brancus ${ }^{5}$, E. Cantoni ${ }^{3,6}$, A. Chiavassa ${ }^{3}$, F. Cossavella ${ }^{4}$, K. Daumiller ${ }^{1}$, V. de Souza ${ }^{7}$, F. Di Pierro ${ }^{3}$, P. Doll ${ }^{1}$, R. Engel ${ }^{1}$, D. Fuhrmann ${ }^{8}$, H.J. Gils ${ }^{1}$, R. Glasstetter ${ }^{8}$, C. Grupen ${ }^{9}$, A. Haungs ${ }^{1}$, D. Heck ${ }^{1}$, J.R. Hörandel ${ }^{10}$, D. Huber ${ }^{4}$, T. Huege ${ }^{1}$, K.-H. Kampert ${ }^{8}$, D. Kang ${ }^{4}$, H.O. Klages ${ }^{1}$, K. Link ${ }^{4}$, P. Łuczak ${ }^{11}$, H.J. Mathes ${ }^{1}$, H.J. Mayer ${ }^{1}$, J. Milke ${ }^{1}$, B. Mitrica ${ }^{5}$, C. Morello ${ }^{6}$, J. Oehlschläger ${ }^{1}$, S. Ostapchenko ${ }^{12}$, N. Palmieri ${ }^{4}$, T. Pierog ${ }^{1}$, H. Rebel ${ }^{1}$, M. Roth ${ }^{1}$, H. Schieler ${ }^{1}$,S. Schoo ${ }^{1}$, F.G. Schröder ${ }^{1}$, O. Sima ${ }^{13}$, G. Toma ${ }^{5}$, G.C. Trinchero ${ }^{6}$, H. Ulrich ${ }^{1}$, A. Weindl ${ }^{1}$, J. Wochele ${ }^{1}$, J. Zabierowski ${ }^{11}$ - KASCADE-Grande Collaboration

${ }^{1}$ Institut für Kernphysik, KIT - Karlsruhe Institute of Technology, Germany

${ }^{2}$ Universidad Michoacana, Inst. Física y Matemáticas, Morelia, Mexico

${ }^{3}$ Dipartimento di Fisica, Università degli Studi di Torino, Italy

${ }^{4}$ Institut für Experimentelle Kernphysik, KIT - Karlsruhe Institute of Technology, Germany

${ }^{5}$ Horia Hulubei National Institute of Physics and Nuclear Engineering, Bucharest, Romania

${ }^{6}$ Osservatorio Astrofisico di Torino, INAF Torino, Italy

${ }^{7}$ Universidade São Paulo, Instituto de Física de São Carlos, Brasil

${ }^{8}$ Fachbereich Physik, Universität Wuppertal, Germany

${ }^{9}$ Department of Physics, Siegen University, Germany

${ }^{10}$ Dept. of Astrophysics, Radboud University Nijmegen, The Netherlands

${ }^{11}$ National Centre for Nuclear Research, Department of Astrophysics, Lodz, Poland

${ }^{12}$ Frankfurt Institute for Advanced Studies (FIAS), Frankfurt am Main, Germany

${ }^{13}$ Department of Physics, University of Bucharest, Bucharest, Romania

E-mail: alexandru.gherghelenipne.ro 
In previous studies based on CORSIKA EAS simulations with the QGSJet-II-02 hadronic interaction model, the observables $\mathrm{S}(200)$ and $\mathrm{S}(500)$ (the charged particle densities at $200 \mathrm{~m}$ and 500 $\mathrm{m}$ from the shower axis) were found to be good candidates for mass discrimination and energy estimation. In order to study the effects of new hadronic interaction models on the reconstruction of EAS from the KASCADE-Grande experiment, a set of CORSIKA simulated showers was computed using the QGSJet-II.04 and EPOS-LHC models. Lateral Energy Correction Functions (LECF) are obtained for the Grande array using a Geant4 simulation code containing a realistic geometry of the Grande station. The LECFs are used to reconstruct the charged particle densities from the energy deposits in the Grande stations. We are using the real and reconstructed lateral distributions of charged particles in order to find corresponding observables for mass discrimination and energy estimation.

The 34th International Cosmic Ray Conference, 30 July- 6 August, 2015

The Hague, The Netherlands

${ }^{*}$ Speaker. 


\section{Introduction}

In parallel to the $N_{\mu}-N_{c h}$ energy and mass estimation procedure used in the KASCADEGrande Experiment [1], the study of the lateral density of charged secondary particles from an EAS can provide sufficient information in order to be used in the development of an alternative reconstruction method. From the analysis of CORSIKA [2] EAS simulations based on the QGSJetII.02 hadronic interaction model, the charged particle densities at $500 \mathrm{~m}(\mathrm{~S}(500)$ parameter[3]) and $200 \mathrm{~m}(\mathrm{~S}(200)$ parameter $[4,5])$ from the shower core, were proven to be good candidates for energy estimation and mass discrimination.

Due to the difficulties in preparing a large number of CORSIKA simulations at energies greater than $10^{16} \mathrm{eV}$ (computing time, size of the output files), and shower fluctuations, it is a challenge to have good statistics. A set of CORSIKA simulations for proton, iron and carbon primaries (180 events for each) with $\theta=20^{\circ} \pm 2^{\circ}$ and energy range of $E_{0}=10^{16}-10^{17} \mathrm{eV}$ was computed for the study of the lateral density of the charged secondary particles. The QGSJet-II.04 and EPOSLHC hadronic interaction models were used in order to test the validity of the $S(500)$ and $S(200)$ observables in the context of the new hadronic interaction models.

\section{S(500), still a good observable for energy estimation}

By comparing the charged particle density for the two hadronic interaction models in the 400 $\mathrm{m}-600 \mathrm{~m}$ range, it was found that the charged particle density at $500 \mathrm{~m}$ practically does not depend on the mass of the primary particle.

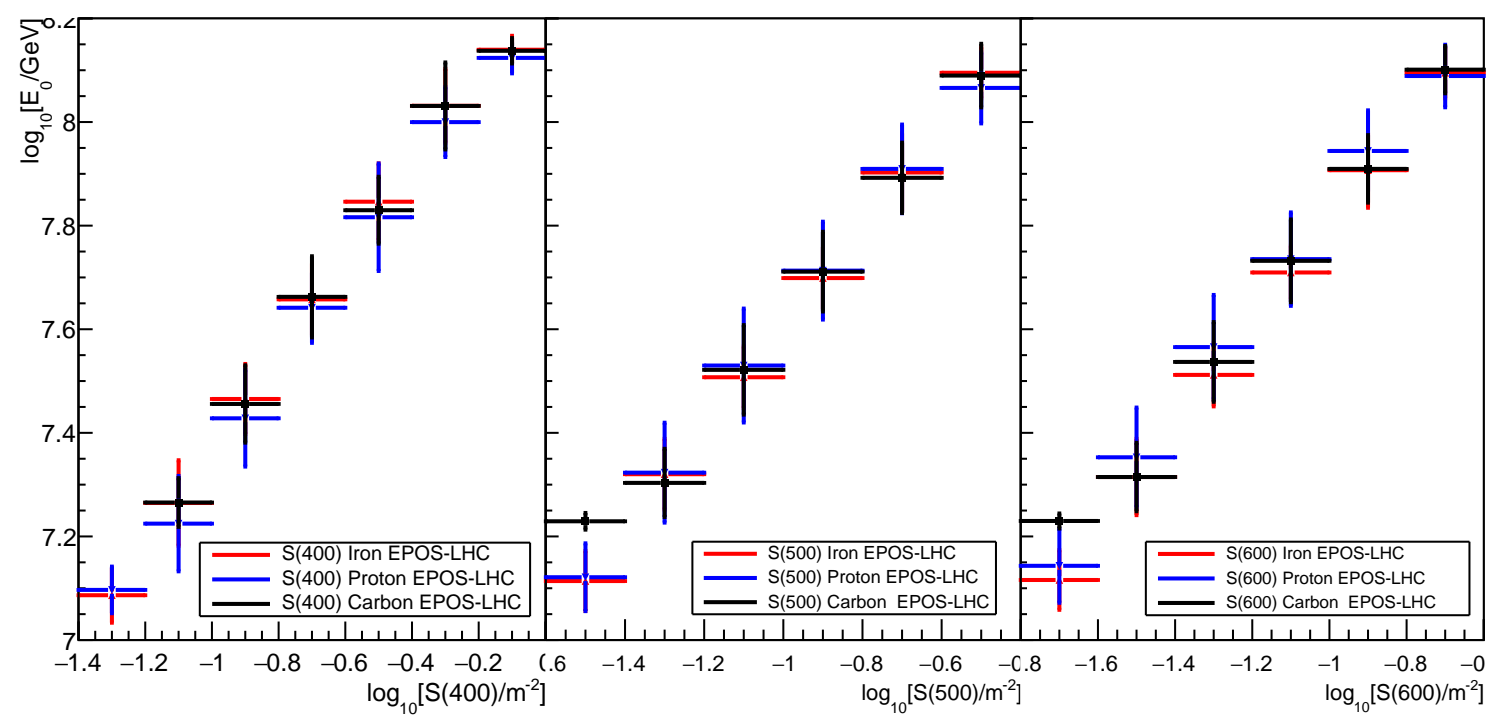

Figure 1: The charged particle density at $400 \mathrm{~m}$ (left), $500 \mathrm{~m}$ (center) and $600 \mathrm{~m}$ (right) for EPOS-LHC simulations for proton, carbon and iron primary particles

If we compare the averaged $\mathrm{S}(500)$ values for the two hadronic interaction models (Fig.3), we observe that the number of charged particles produced using the EPOS-LHC model is slightly 


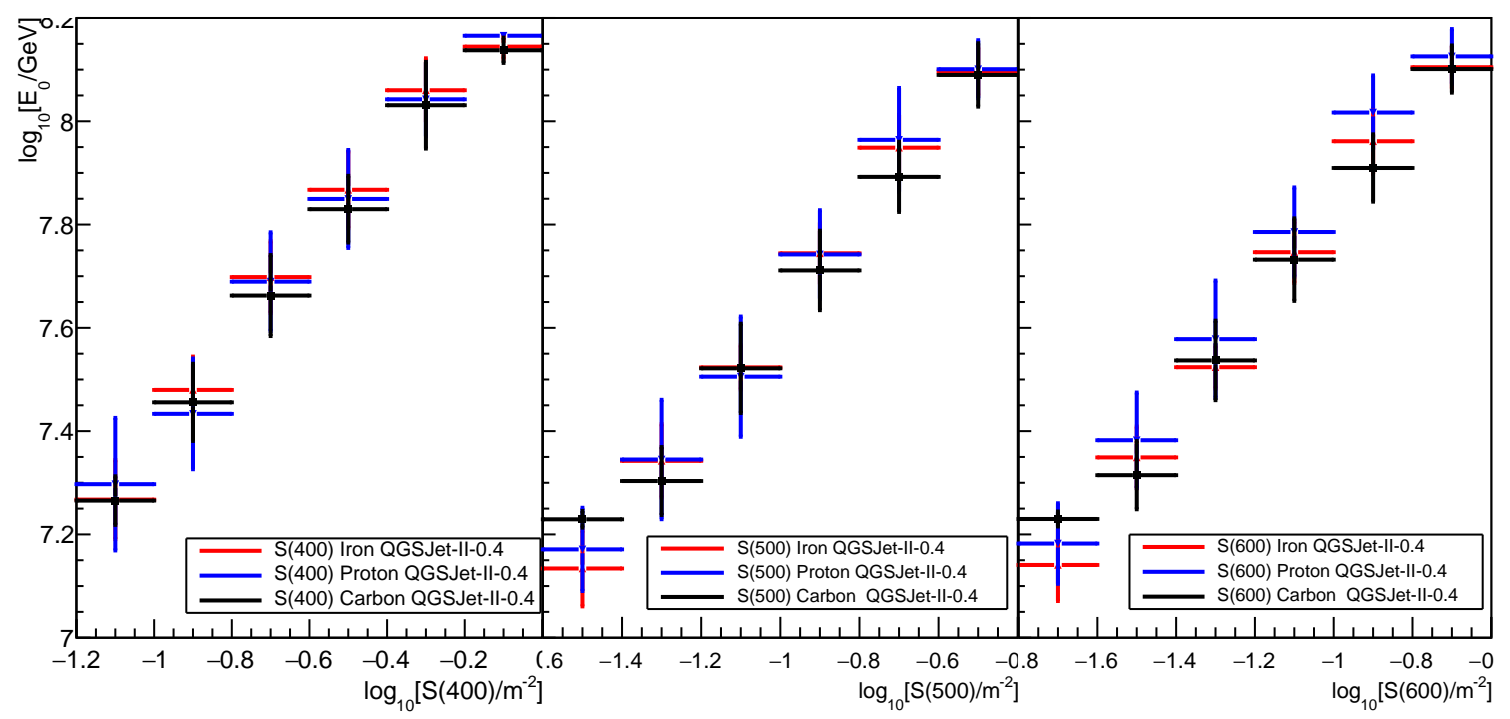

Figure 2: The charged particle density at $400 \mathrm{~m}$ (left), $500 \mathrm{~m}$ (center) and $600 \mathrm{~m}$ (right) for QGSJet-II-04 simulations

greater than the one obtained with QGSJet-II.04. As a result, a higher energy will be reconstructed from the same experimental data using QGSJet-II.04 than using EPOS-LHC. These effects will be the subject of future work.

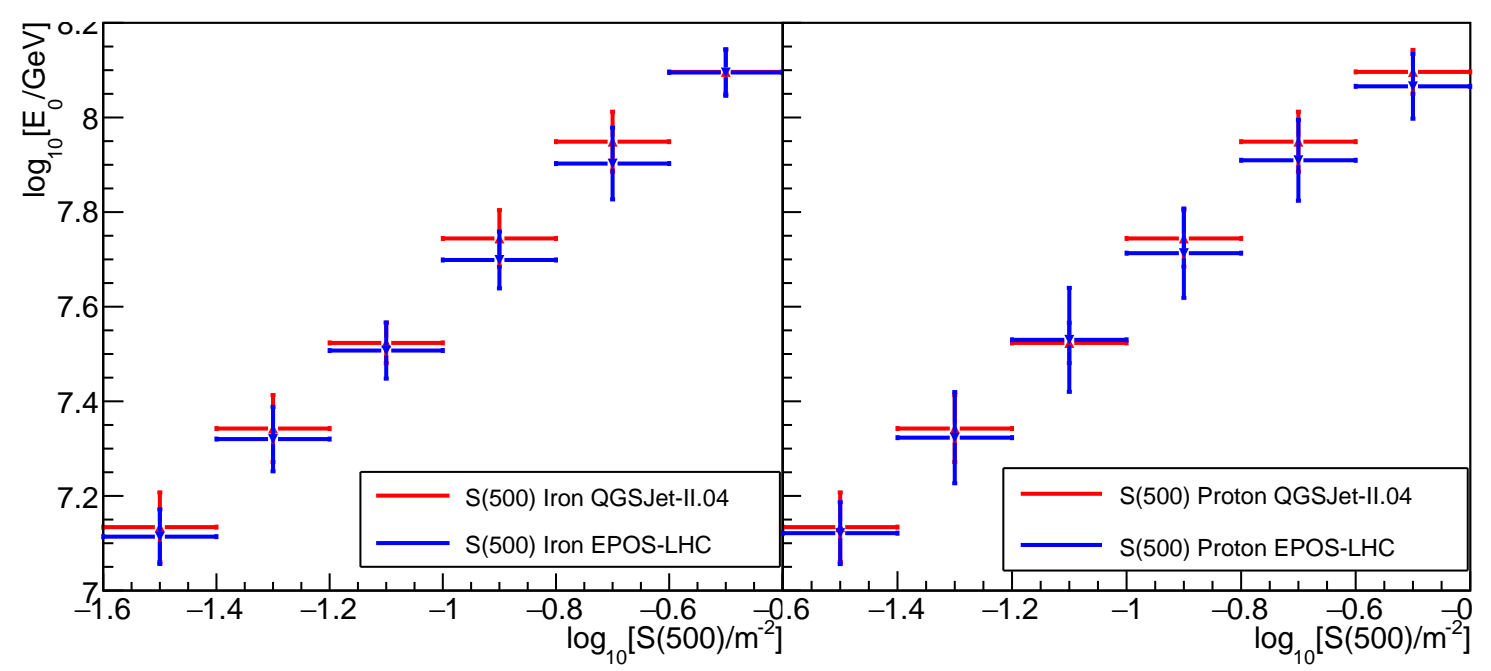

Figure 3: The charged particle density at $500 \mathrm{~m}$ for EPOS-LHC and QGSJet-II.04 simulations

\section{3. $S(200)$ - An observable of mass discrimination}

Previous studies of CORSIKA simulations (using the QGSJet-I hadronic interaction model) showed that the charged particle density close to the shower core is mass dependent. The charged 
particle density at $100 \mathrm{~m}, 200 \mathrm{~m}$ and $300 \mathrm{~m}$ from the shower core obtained for the new set of simulations is presented in Fig.4 and Fig.5.

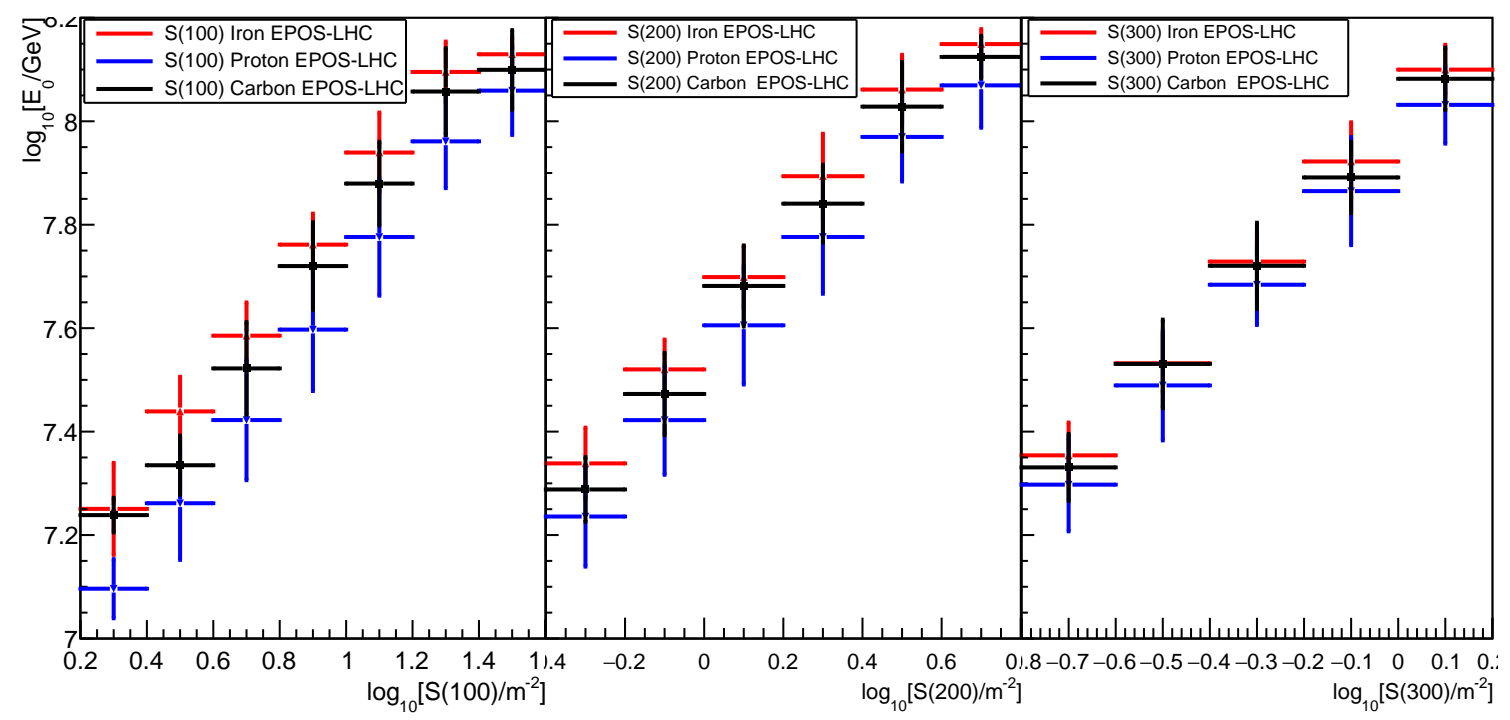

Figure 4: The charged particle density at $100 \mathrm{~m}$ (left), $200 \mathrm{~m}$ (center) and $300 \mathrm{~m}$ (right) for EPOS-LHC simulations

Due to the large number of charged particles in the region of $100 \mathrm{~m}$ from the shower core (in particular for EAS with $E_{0}>10^{17} \mathrm{eV}$ ) detector saturation may occur. For that reason, the charged particle density at $200 \mathrm{~m}$ from the shower core ( $\mathrm{S}(200)$ observable) seems to be a good candidate for mass discrimination.

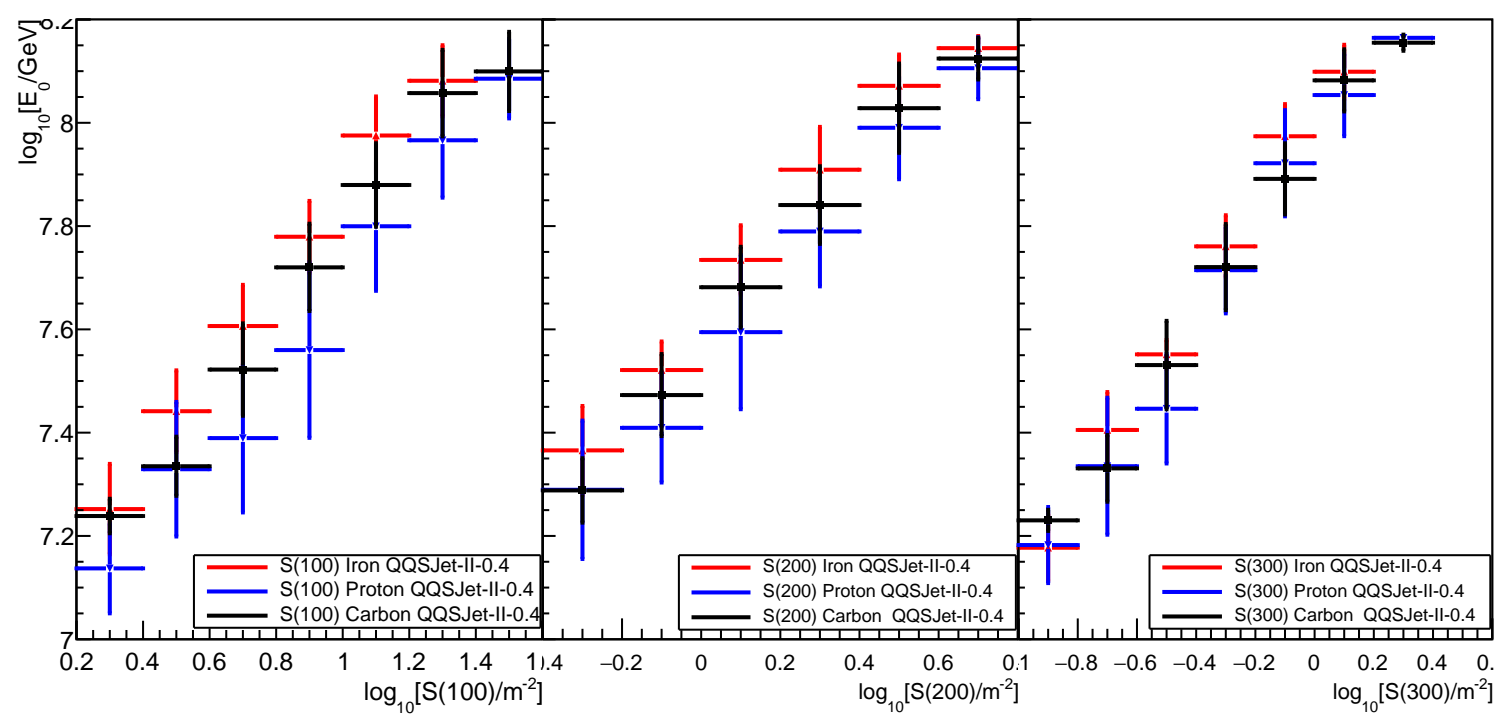

Figure 5: The charged particle density at $100 \mathrm{~m}$ (left), $200 \mathrm{~m}$ (center) and $300 \mathrm{~m}$ (right) for QGSJet-II-04 simulations 


\section{Perspectives}

The experimental energy deposits measured with the Grande array are converted into charged particle densities using the Lateral Energy Correction Function (LECF). The LECF represents the energy deposited in the Grande station by all the secondary particles in a shower divided by the number of charged particles and calculated for the position of the detector relative to the shower core.

The standard reconstruction procedure uses a fixed, mean value for the LECF (Fig.6), and does not include corrections for different energies and incidence angles of the secondary shower particles interacting with the detectors. The standard LECF was computed using GEANT3 and a schematic geometry of the Grande stations.

We intend to obtain more realistic LECF functions including all the details of the Grande station (Fig.7) in simulations carried out with Geant4.10 [6]. The distribution of the secondary particles impinging on the stations will be obtained using CORSIKA simulations including QGSJetII.04 and EPOS-LHC. In order to take into account the azimuth dependence of the LECF, a very dense array of Grande stations ( $800 \times 800 \mathrm{~m}^{2}$ with $10 \mathrm{~m}$ distance between stations) will be implemented in the simulation.

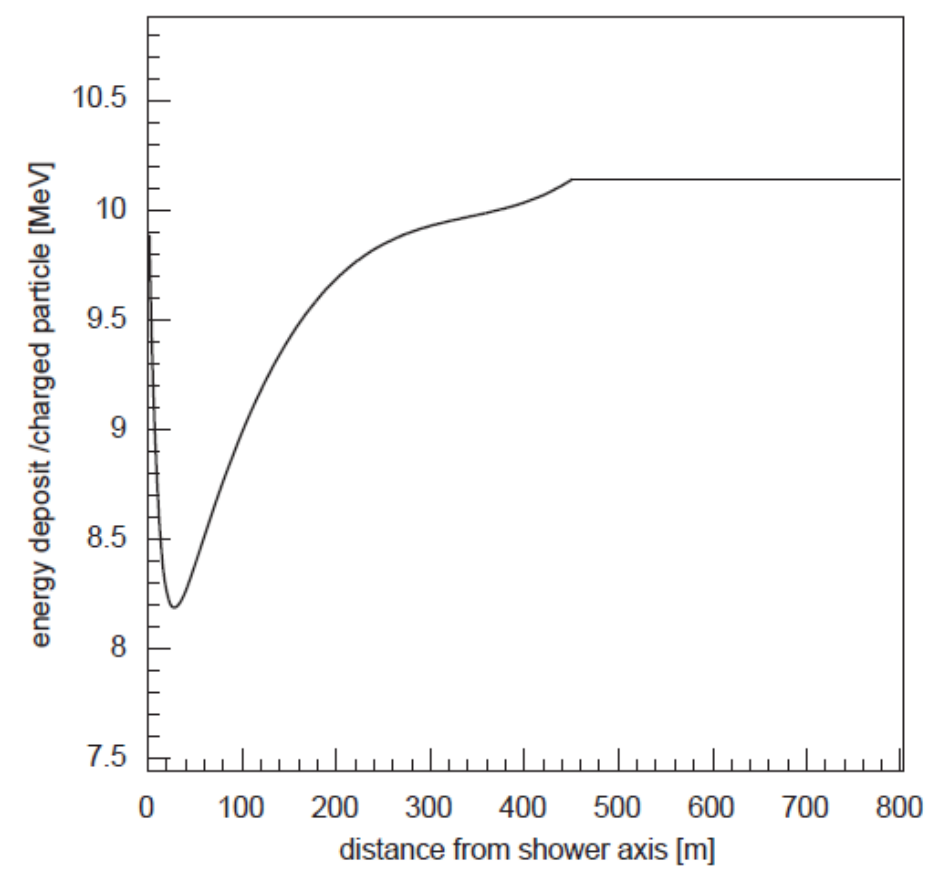

Figure 6: Standard LECF used at the KASCADE-Grande experiment

The set of LECFs that will be obtained will be used in the reconstruction of the charged particle density for simulated and experimental data. The primary particle's energy and mass will be evaluated using the $S(500)$ and $S(200)$ observable.

\section{Acknowledgment}

The authors would like to thank the members of the engineering and technical staff of the KASCADEGrande collaboration, who contributed to the success of the experiment. The KASCADE-Grande 


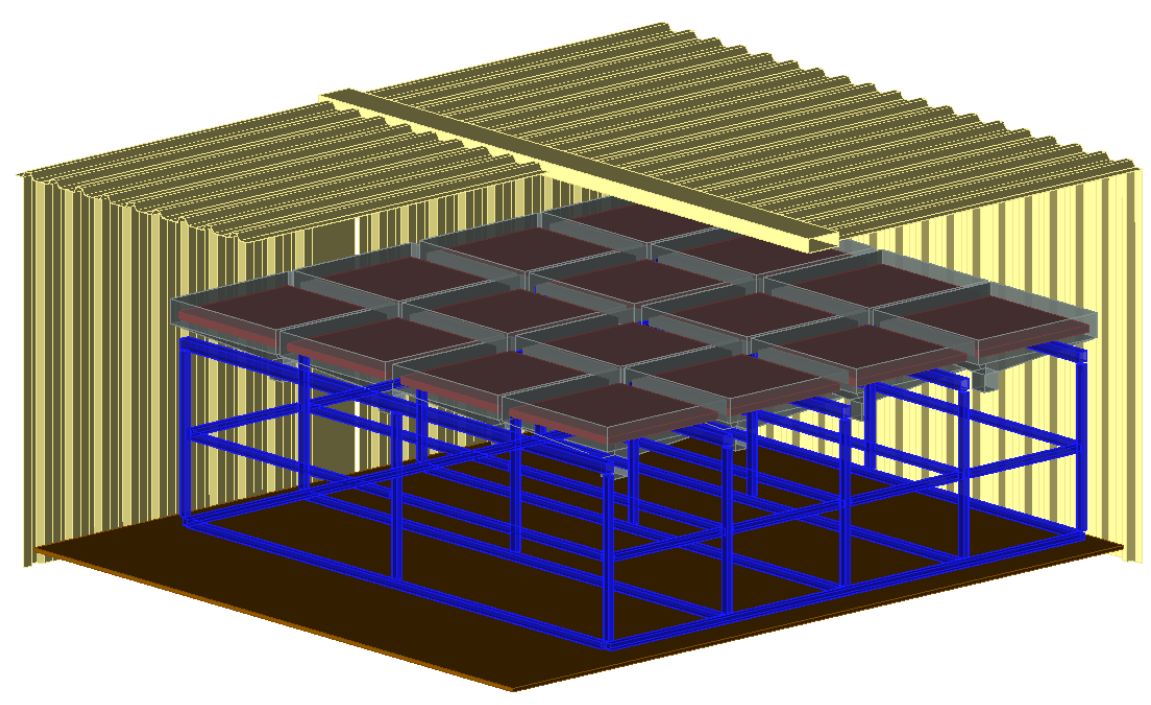

Figure 7: Grande station geometry used in the Geant 4 simulation

experiment is supported in Germany by the BMBF and by the 'Helmholtz Alliance for Astroparticle Physics - HAP' funded by the Initiative and Networking Fund of the Helmholtz Association, by the MIUR and INAF of Italy, the Polish Ministry of Science and Higher Education, and the Romanian Authority for Scientific Research UEFISCDI (PNII-IDEI grants 271/2011 and 17/2011). J.C.A.V. acknowledges the partial support of CONACyT.

\section{References}

[1] Apel W D et al., The KASCADE-Grande experiment, NIM A $\mathbf{6 2 0}$ (2010) 202

[2] D. Heck et al.,Report FZKA,6019 (1998), Forschungszentrum Karlsruhe

[3] G.Toma et al., Romanian Reports in Physics, 63 (2011) 383-393

[4] H. Rebel, O. Sima et al. KASCADE-Grande collaboration, Proc. 29th ICRC Pune India, 6 (2005) 297;

[5] I.M. Brancus et al. KASCADE-Grande collaboration, Proc. 29th ICRC Pune India, 6 (2005) 361

[6] S. Agostinelli for the Geant4 Collaboration NIM A, 506 (2003) 250-303 